Caracterização Físico-Química de Complexos de Inclusão Contendo os Monoterpenos Cânfora e 1,8-Cineol, Constituintes Majoritários dos Óleos Essenciais de Croton conduplicatus, em $\beta$-Ciclodextrina

\author{
Alves, C. S. C.; Oliveira, A. P.; Oliveira-Junior, R. G.; Santos, R. F.; Santos, \\ A. D. C.; Tavares; G. F.; Almeida, J. R. G. S.* \\ Rev. Virtual Quim., 2019, 11 (2), 401-410. Data de publicação na Web: 11 de março de 2019 \\ http://rvq.sbq.org.br

\section{Physico-Chemical Characterization of the Inclusion Complexes Containing the Monoterpenes Camphor and 1,8-Cineol, Major Constituents of the Essential Oils of Croton conduplicatus, in $\beta$-Cyclodextrin}

\begin{abstract}
Cyclodextrins (CDs) constitute a class of pharmaceutical excipients composed of $D$-glucopyranose units. Through the formation of inclusion complexes with CDs it is possible to administer nonpolar substances, such as monoterpenes, and evaluate their pharmacological activity. Considering the relevance of the discovery of new drugs, as well as the need to develop new pharmaceutical forms, this study aimed to develop inclusion complexes of monoterpenes contained in the essential oils of leaves and stem bark of Croton conduplicatus in $\beta$-cyclodextrin. The complexes were prepared and characterized by nuclear magnetic resonance (NMR), Fourier-transform infrared spectroscopy (FT-IR) and scanning electron microscopy (SEM). The analysis allowed us to conclude that there was in fact the formation of the inclusion complexes of camphor and 1,8-cineole in $\beta$-cyclodextrin, and the results were important to complement the studies being carried out in the area of pharmaceutical technology.
\end{abstract}

Keywords: Euphorbiaceae; Croton conduplicatus; cyclodextrins; nuclear magnetic resonance.

\title{
Resumo
}

As ciclodextrinas (CDs) constituem uma classe de excipientes farmacêuticos compostas por unidades de $D$-glicopiranose. Através da formação de complexos com essas moléculas, pode-se administrar substâncias apolares, como os monoterpenos, e avaliar a sua atividade farmacológica. Considerando a importância da descoberta de novos fármacos bem como a necessidade de desenvolvimento de novas formas farmacêuticas, este estudo visou desenvolver complexos de inclusão de dois monoterpenos contidos nos óleos essenciais das folhas e cascas do caule de Croton conduplicatus em $\beta$-ciclodextrina. Os complexos foram preparados e caracterizados por ressonância magnética nuclear (RMN), espectroscopia de infravermelho com transformada de Fourier (FT-IR) e microscopia eletrônica de varredura (MEV). A análise permitiu concluir que houve de fato a formação dos complexos de inclusão de cânfora e 1,8-cineol em $\beta$-ciclodextrina, e os resultados foram importantes para complementar os estudos que vêm sendo realizados na área de tecnologia farmacêutica.

Palavras-chave: Euphorbiaceae; Croton conduplicatus; ciclodextrinas; ressonância magnética nuclear.

\footnotetext{
* Universidade Federal do Vale do São Francisco, Núcleo de Estudos e Pesquisas de Plantas Medicinais (NEPLAME), CEP 56304-205, Petrolina-PE, Brazil.

M jackson.guedes@univasf.edu.br DOI: $\underline{10.21577 / 1984-6835.20190030}$
} 


\title{
Caracterização Físico-Química de Complexos de Inclusão Contendo os Monoterpenos Cânfora e 1,8-Cineol, Constituintes Majoritários dos Óleos Essenciais de Croton conduplicatus, em $\beta$-Ciclodextrina
}

\author{
Cristiane dos S. C. Alves, a Ana Paula de Oliveira, a Alan Diego da C. \\ Santos, ${ }^{\text {a }}$ Raira F. dos Santos, ${ }^{\text {a }}$ Raimundo G. de Oliveira Junior, ${ }^{\text {a }}$ Ginetton \\ F. Tavares, ${ }^{\mathrm{b}}$ Jackson R. G. da S. Almeida ${ }^{\mathrm{a}, *}$ \\ ${ }^{a}$ Universidade Federal do Vale do São Francisco, Núcleo de Estudos e Pesquisas de Plantas \\ Medicinais (NEPLAME), CEP 56304-205, Petrolina-PE, Brazil. \\ ${ }^{b}$ Universidade Federal do Vale do São Francisco, Instituto de Pesquisa em Ciência dos \\ Materiais (IPCM), CEP 48902-300, Juazeiro-BA, Brazil. \\ *jackson.guedes@univasf.edu.br
}

Recebido em 6 de fevereiro de 2019. Aceito para publicação em 6 de fevereiro de 2019

1. Introdução

2. Parte Experimental

2.1. Preparação dos complexos de inclusão

2.2. Caracterização físico-química dos complexos de inclusão e das misturas físicas

\section{Resultados e Discussão}

3.1. Caracterização por espectroscopia de infravermelho com transformada de Fourier (FT-IR)

3.2. Caracterização por microscopia eletrônica de varredura (MEV)

3.3. Caracterização por ressonância magnética nuclear (RMN)

\section{Conclusão}

\section{Introdução}

A flora do semiárido nordestino brasileiro apresenta uma impressionante variedade de espécies vegetais, que contêm compostos terapêuticos de interesse para a biotecnologia farmacêutica. ${ }^{1}$ Inserida nessa biodiversidade, a família Euphorbiaceae é uma das mais extensas famílias botânicas, compreendendo cerca de 300 gêneros e 7600 espécies, dentre eles o gênero Croton. ${ }^{1,2}$

Com cerca de 1.200 espécies, Croton é o segundo maior gênero da família Euphorbiaceae. Tem distribuição pantropical, embora a maioria dos seus representantes ocorra nas Américas. Na América do Sul, o Brasil é o país que congrega o maior número 
de espécies, aproximadamente 350, amplamente distribuídas nos mais diversos ambientes, destacando-se o Cerrado, a Caatinga e os Campos Rupestres. Na região Nordeste do Brasil, as espécies de Croton são conhecidas popularmente como velames ou marmeleiros, e são usadas para os mais diversos fins, inclusive para uso medicinal. ${ }^{3}$

Este gênero contém um complexo arsenal bioquímico, possuindo expressiva relevância econômica, alicerçada em seu conteúdo de óleos essenciais e diversas substâncias ativas como alcalóides, fenilpropanoides, monoterpenos, sesquiterpenos, diterpenos e lignanas. ${ }^{4}$ Muitas espécies deste gênero são produtoras de óleos essenciais, distribuídos em todos os órgãos da planta, principalmente nas folhas e nas cascas do caule. ${ }^{1,2}$

Várias atividades biológicas, incluindo antiinflamatória, antinociceptiva, gastroprotetora e sobre o sistema cardiovascular foram relatadas para o óleo essencial de várias espécies deste gênero em modelos animais. ${ }^{5}$

A espécie Croton conduplicatus é conhecida popularmente na região Nordeste do Brasil como quebra faca. Estudos anteriores realizados pelo nosso grupo de pesquisa têm mostrado a composição química do óleo essencial das cascas do caule $^{6}$ e folhas ${ }^{7}$ desta espécie, cuja composição química é caracterizada pela presença de mono e sesquiterpenos, dentre eles, cânfora e 1,8-cineol. Estes óleos essenciais têm demonstrado atividade antinociceptiva ${ }^{8}$ como também ansiolítica e sedativa. ${ }^{9}$

Apesar das atividades biológicas, a baixa solubilidade em água, juntamente com a volatilidade, a instabilidade, a meia-vida curta e a toxicidade prejudicam o uso de óleos essenciais e seus constituintes químicos no desenvolvimento de preparações farmacêuticas ou cosméticas. A partir disso, uma das principais abordagens atualmente utilizadas é a formação de um complexo de inclusão com ciclodextrinas (CDs), que fornece tanto estabilização quanto liberação controlada de materiais encapsulados. ${ }^{10}$

As CDs obtidas com maior rendimento, conhecidas como naturais, contém seis, sete e oito unidades de glicose, sendo denominadas de $\alpha$-ciclodextrina, $\beta$-ciclodextrina e $\gamma$ ciclodextrina, respectivamente. A estrutura espacial cônica e a orientação dos grupos hidroxílicos para o exterior conferem a estes açúcares cíclicos propriedades físico-químicas únicas, sendo capazes de solubilizar-se em meio aquoso e ao mesmo tempo encapsular no interior da sua cavidade moléculas hidrofóbicas. ${ }^{11}$

Esses complexos de inclusão possuem propriedades físico-químicas e biológicas que são bastante diferentes da substância complexada, podendo ser utilizados para aumentar a solubilidade, diminuir a volatilidade e aumentar a estabilidade das substâncias. ${ }^{10,12}$ O critério essencial é simplesmente que a molécula encapsulada seja de forma e tamanho adequado para entrar na cavidade da molécula hóspede. ${ }^{12}$

O objetivo desse trabalho foi obter complexos de inclusão contendo os monoterpenos cânfora e 1,8-cineol, os quais estão presentes nos óleos essenciais das folhas e cascas do caule de Croton conduplicatus, em $\beta$-ciclodextrina, bem como realizar a sua caracterização físico-química.

\section{Parte Experimental}

\subsection{Preparação dos complexos de inclusão}

Na proporção de 1:1, foram pesados 100,7 $\mathrm{mg}$ de cânfora e $0,764 \mathrm{~g}$ de $\beta$-ciclodextrina e colocados em um erlenmeyer. Para a formação do complexo, à mistura foi adicionada uma solução de $25 \%$ de água e $75 \%$ de etanol, deixando em um agitador magnético por 24 horas. Em seguida, a mistura formada foi liofilizada. Para a formação da mistura física, foram pesados $0,0509 \mathrm{~g}$ de cânfora e $0,37 \mathrm{~g}$ de $\beta$ ciclodextrina, que foram misturados em um gral com pistilo por quatro minutos.

O procedimento anterior foi realizado para o composto 1,8-cineol. No entanto, foram 
utilizados 4,3 mg desse composto e 0,2958 g de $\beta$-ciclodextrina, pesados na proporção de $1: 1$, e para a mistura física foram pesados 0,0135 g de 1,8-cineol e 0,0985 g de $\beta$ ciclodextrina, respectivamente.

2.2. Caracterização físico-química dos complexos de inclusão e das misturas físicas

2.2.1 Caracterização por espectroscopia de infravermelho com transformada de Fourier (FT-IR)

O experimento foi realizado em parceria com o Instituto Federal de Ciência e Tecnologia do Sertão Pernambucano (IFSertão), em Petrolina-PE, em um aparelho PerkinElmer, Versão 10.4.00.

2.2.2 Caracterização por microscopia eletrônica de varredura (MEV)

A superfície dos monoterpenos, das misturas físicas e dos complexos de inclusão foram examinadas em um microscópio eletrônico de varreduta modelo TescanVEGA3. As amostras foram preparadas pela montagem de pós em fita de carbono colocada sob película de alumínio. Depois, os pós foram metalizados com pó de ouro durante 250 segundos e examinados usando MEV a $10 \mathrm{kV}$.

2.2.3 Caracterização por ressonância magnética nuclear (RMN)
Os experimentos de ressonância magnética nuclear $1 \mathrm{D}$ de ${ }^{1} \mathrm{H}$ (RMN de ${ }^{1} \mathrm{H}$ ) e $2 \mathrm{D}$ $\left({ }^{1} \mathrm{H}-{ }^{1} \mathrm{H}\right.$ ROESY) foram adquiridos utilizando $\mathrm{D}_{2} \mathrm{O}$ e conduzidos em espectrômetro Bruker ${ }^{\mathrm{TM}}$ $400 \mathrm{MHz}$ ASCEND III, operando a 9,4 Tesla, observando os núcleos de ${ }^{1} \mathrm{H}$ a $400,13 \mathrm{MHz}$, equipado com sonda de detecção direta de 5 $\mathrm{mm}$ e gradiente de campo no eixo $z$. Os deslocamentos químicos de RMN de ${ }^{1} \mathrm{H}$ foram expressos em relação ao sinal do TMSP em $\delta$ 0,00 (composto de referência) e as constantes de acoplamento $(J)$ foram expressas em $\mathrm{Hz}$.

\section{Resultados e Discussão}

3.1. Caracterização por espectroscopia de infravermelho com transformada de Fourier (FT-IR)

A análise por FT-IR do complexo cânfora/ $\beta$ $C D$ (Figura 1 ) mostrou forte semelhança com o padrão de absorção da $\beta-C D$, assim como redução da intensidade das bandas características da cânfora. Além disso, nota-se claramente as bandas de absorção na região de $3000 \mathrm{~cm}^{-1}$ (estiramento $\mathrm{O}-\mathrm{H}$ ), $2900 \mathrm{~cm}^{-1}$ (estiramento $\mathrm{C}-\mathrm{H}$ ), em $1154 \mathrm{~cm}^{-1}$ (estiramento $\mathrm{C}-\mathrm{O}$ ) e $1026 \mathrm{~cm}^{-1}$ (estiramento $\mathrm{C}-\mathrm{O}-\mathrm{C}$ ), todos característicos da $\beta-C D$, caracterizadas por um leve deslocamento das bandas de estiramento $\mathrm{O}-\mathrm{H}$ e de estiramento $\mathrm{C}-\mathrm{H}$, além da deformação da banda da carbonila $(C=O)$ na região de $1720 \mathrm{~cm}^{-1}$ e da banda na região de $2960 \mathrm{~cm}^{-1}$ (estiramento $\mathrm{C}-\mathrm{H}$ ), representativas da cânfora. Esse padrão de absorção não é observado na mistura física nem é verificada a banda da carbonila, não deformada como na formação do complexo, havendo uma espécie de sobreposição dos sinais de ambos, sugerindo pouca interação entre eles. 


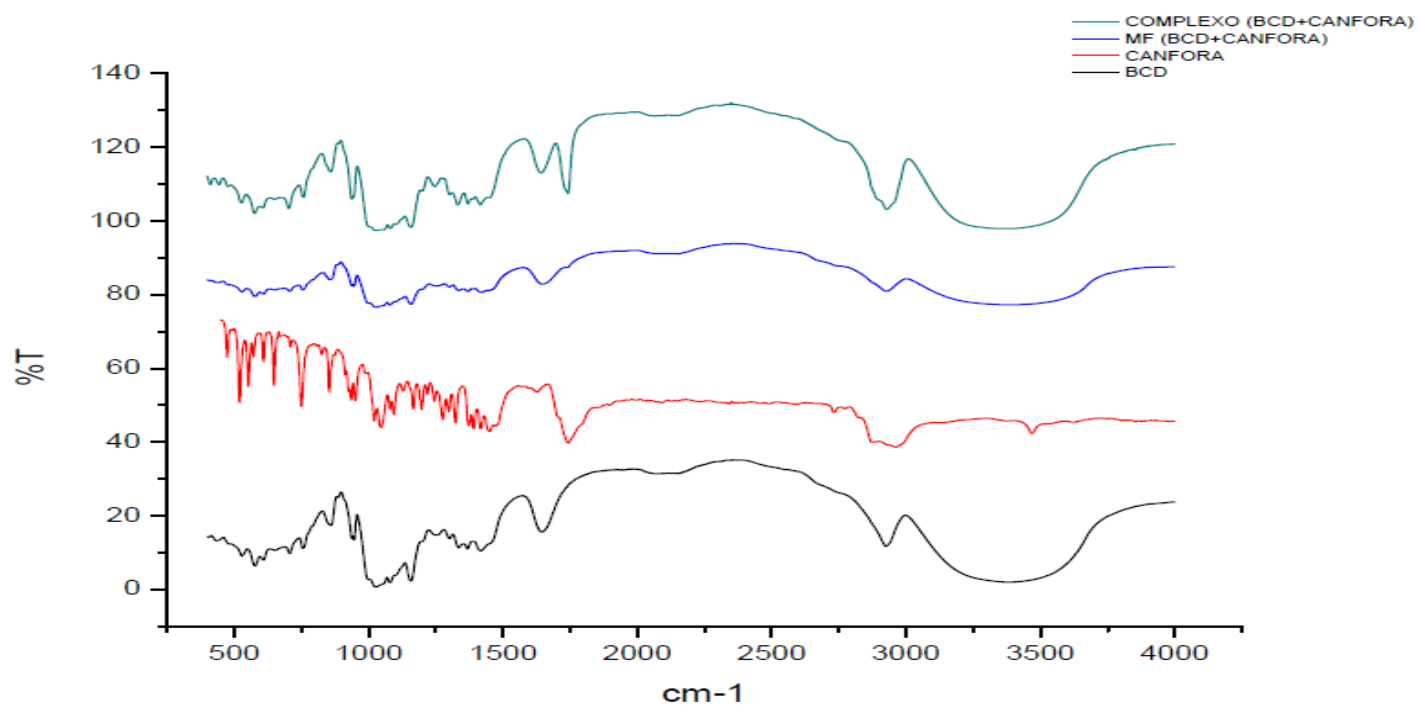

Figura 1. Caracterização por FT-IR do complexo de inclusão de cânfora em $\beta$-ciclodextrina (em verde); para a mistura física de cânfora e $\beta$-ciclodextrina (em azul); para a cânfora (em vermelho); para a $\beta$-ciclodextrina (em preto)

Da mesma forma que o padrão de absorção do complexo de inclusão de cânfora, o complexo 1,8-cineol/B-CD (Figura 2) apresentou deslocamento das bandas e sua posterior deformação com a formação do complexo. As bandas características do 1,8cineol em $2917 \mathrm{~cm}^{-1}$ e em $1464 \mathrm{~cm}^{-1}$ (estiramento $\mathrm{C}-\mathrm{H})$ foram imensamente reduzidas e deformadas. Todas essas informações ajudam a comprovar o fato de que houve a formação do complexo, visto que a inclusão de uma molécula na cavidade da $\beta$ $C D$ gera uma restrição conformacional, reduzindo a livre movimentação das moléculas inclusas e, assim, reduzindo a intensidade do sinal.

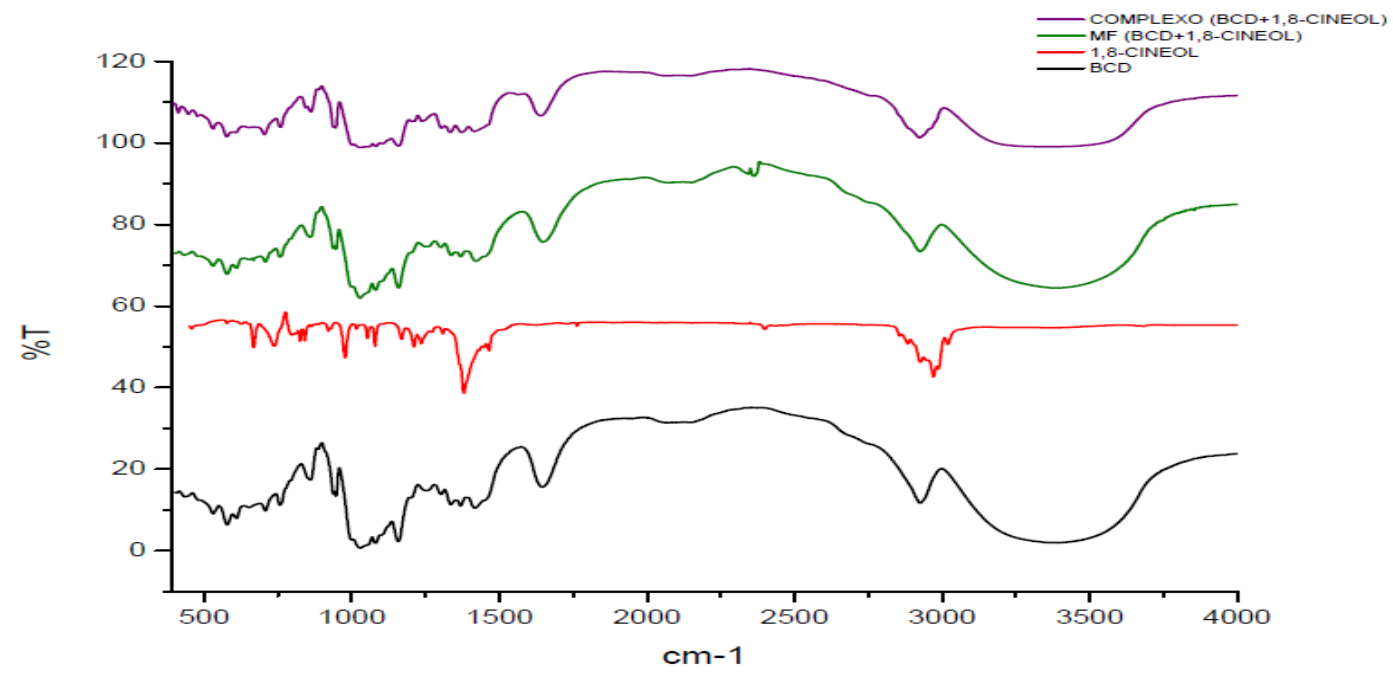

Figura 2. Caracterização por FT-IR do complexo de inclusão de 1,8-cineol em $\beta$-ciclodextrina (em roxo); para a mistura física de 1,8-cineol e $\beta$-ciclodextrina (em verde); para o 1,8-cineol (em vermelho); para a $\beta$-ciclodextrina (em preto) 
3.2. Caracterização por microscopia eletrônica de varredura (MEV)

A análise das imagens da microscopia da $\beta$ ciclodextrina a caracterizaram como uma morfologia em blocos retangulares bastante evidentes. Na mistura física (MF) com 1,8cineol, o que se observa é a existência de um grande bloco intacto de $\beta$-ciclodextrina e uma outra morfologia atribuída ao 1,8-cineol, comprovando que na MF ambos continuam existindo na sua forma original, não formando um complexo entre si. Diferentemente da MF, nas imagens que correspondem à amostra que passou pela metodologia de formação do complexo, nota-se o aparecimento de uma estrutura única em formato menor que os blocos e em forma de raspas, e não se observa a existência do pó que existia na MF. Devido à tendência de estar no estado líquido e volatilizar à temperatura ambiente, não foi possível realizar a análise do 1,8-cineol isolado por MEV.

A análise das imagens da cânfora permitiu caracterizá-la morfologicamente como tendo um aspecto irregular amorfo, possuindo estrutura semelhante a fios irregulares. A MF aponta a existência de blocos uniformes e intactos, característicos da $\beta$-ciclodextrina, e partes evidentes de estruturas semelhantes a fios, características da cânfora. Assim como na formação do complexo de 1,8-cineol com $\beta$ ciclodextrina, podem ser vistas mudanças na conformação, de blocos para partículas menores e sem a presença característica da cânfora. As mudanças morfológicas observadas são mais um indicativo de que realmente houve a formação de um complexo de inclusão.

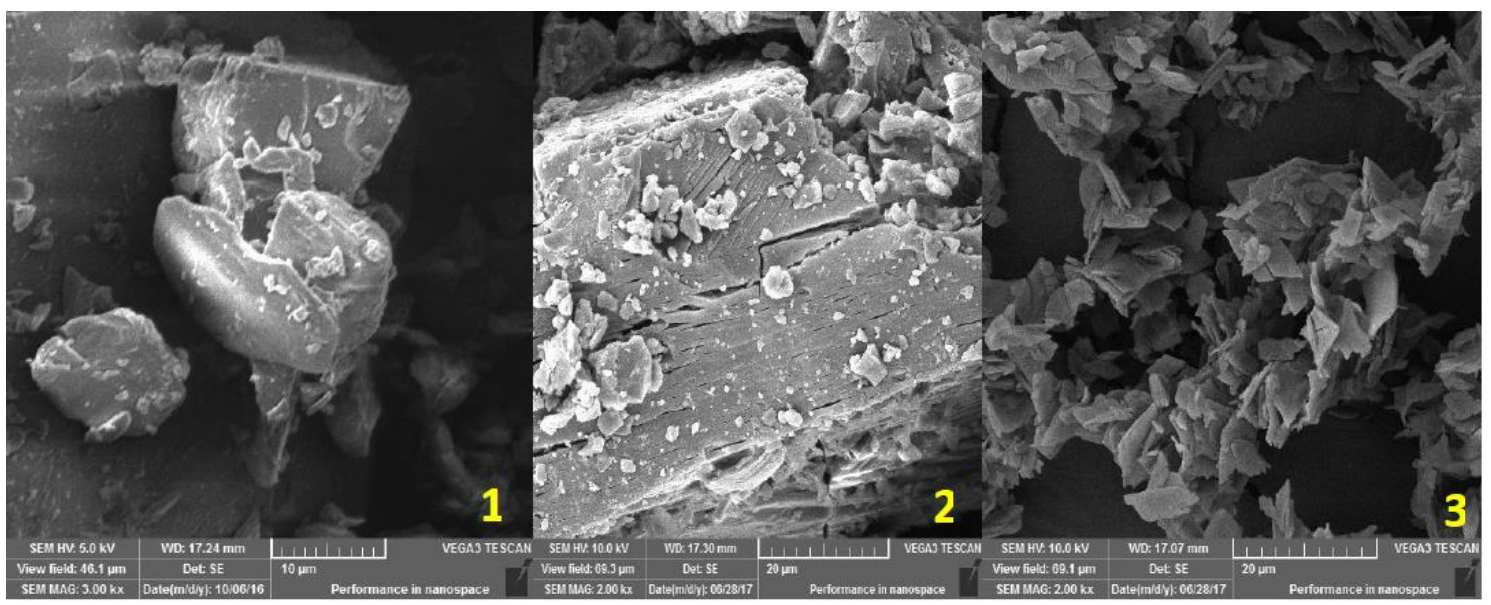

Figura 3. Microscopia eletrônica de varredura de (1) $\beta$-CD; (2) mistura física de 1,8-cineol/ $\beta$-CD e (3) do complexo de inclusão de 1,8-cineol/ $\beta$-CD. Aumentos de 3000x, 2000x e 3000x, respectivamente 


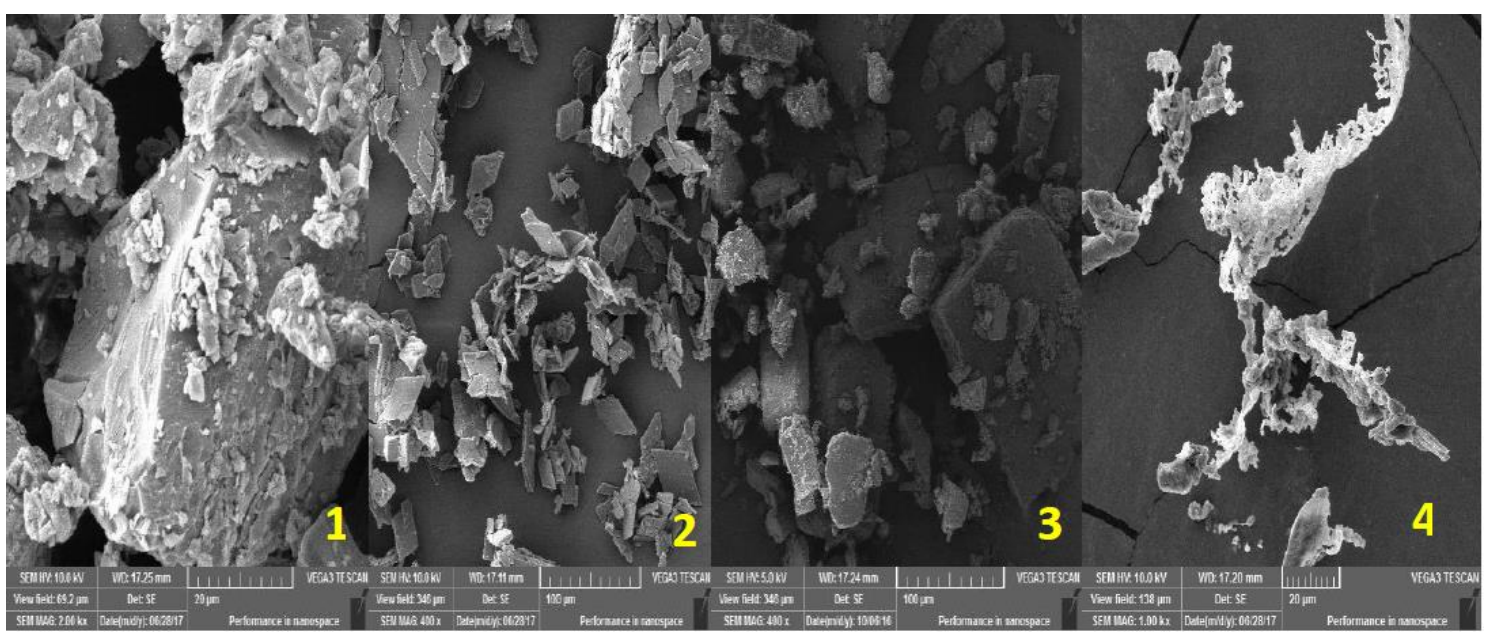

Figura 4. Microscopia eletrônica de varredura de (1) mistura física de cânfora/ $\beta-C D$; $(2)$ do complexo de inclusão de cânfora/ $\beta-C D$; (3) da $\beta-C D$; (4) da cânfora. Aumentos de 2000x, 400x, $400 x$ e $1000 x$, respectivamente

\subsection{Caracterização por ressonância magnética nuclear (RMN)}

A formação dos complexos de inclusão foi observada a partir dos deslocamentos químicos dos prótons da $\beta$-ciclodextrina que ficam para o interior da cavidade, os hidrogênios H-3 e H-5, que irão ser alterados, uma vez que a molécula visitante estiver dentro dela. Conforme o esperado, o experimento de $\mathrm{RMN}$ de ${ }^{1} \mathrm{H}$ demonstrou que os hidrogênios $\mathrm{H}-3$ e $\mathrm{H}-5$ se mostraram em uma região de menor frequência ao formar um complexo de inclusão com os monoterpenos, indicando que foram blindados na presença da molécula visitante. No complexo de inclusão de cânfora em $\beta$ ciclodextrina, o que se observa é que o $\mathrm{H}-3$ está interagindo mais fortemente com a molécula visitante, enquanto no complexo de inclusão de 1,8-cineol em $\beta$-ciclodextrina, o que se observa é que $\mathrm{H}-5$ interage mais fortemente com a molécula visitante, e isso pode indicar o modo como ocorre a inclusão da molécula na $\beta$-ciclodextrina. Esses deslocamentos químicos podem ser observados nas Tabelas 1 e 2, abaixo.

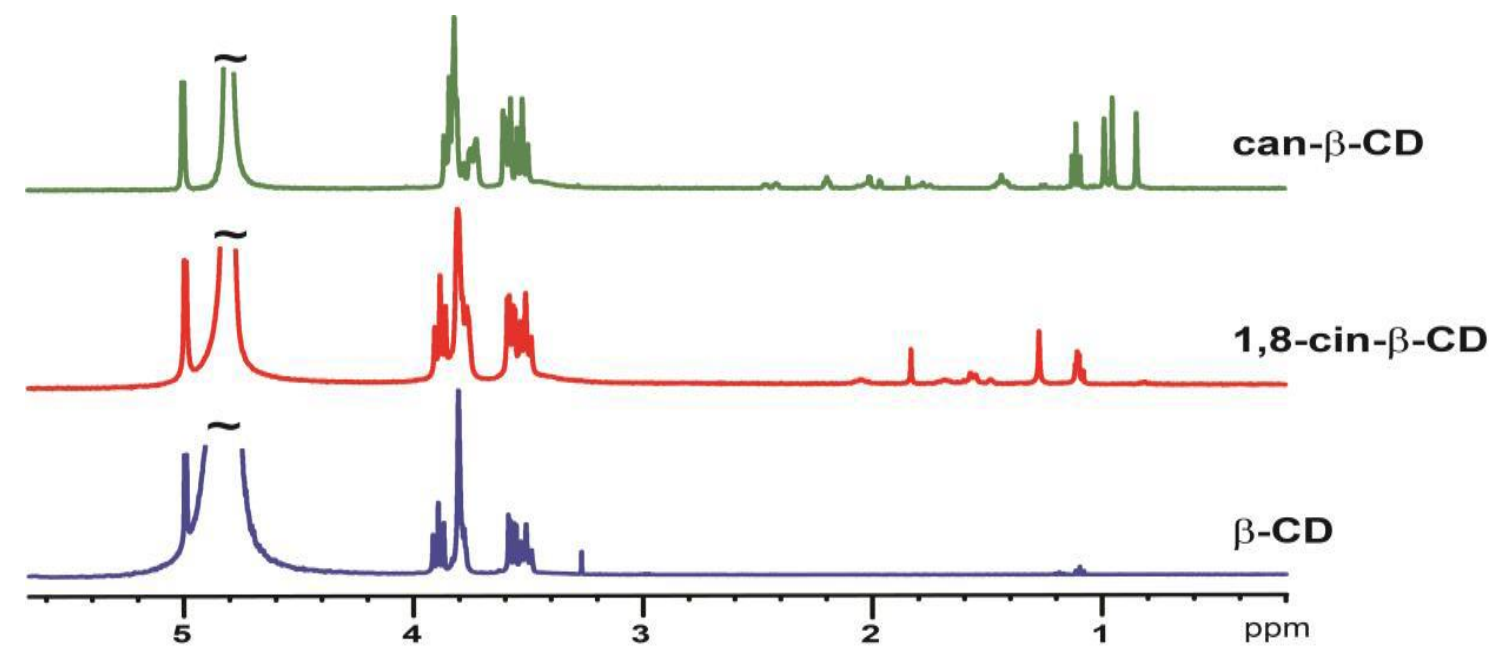

Figura 5. Experimento de $\mathrm{RMN}$ de ${ }^{1} \mathrm{H}$ em $\mathrm{D}_{2} \mathrm{O}$ para a $\beta$-ciclodextrina sozinha (em azul); em um complexo de inclusão com 1,8-cineol (em vermelho); em um complexo de inclusão com cânfora (em verde) 
Tabela 1. Deslocamentos químicos $(\delta)$ dos prótons de $\beta-C D$ complexada com 1,8-cineol em $\mathrm{D}_{2} \mathrm{O}$

\begin{tabular}{cccc}
\hline Próton & $\boldsymbol{\beta}-\mathrm{CD}$ & $\boldsymbol{\beta}-\mathrm{CD} / \mathbf{1 , 8 - \text { cineol }}$ & $\boldsymbol{\Delta} \boldsymbol{\delta}$ \\
\hline H-1 & 4.99060 & 4.98880 & 0.002 \\
H-2 & 3.56900 & 3.57003 & 0.001 \\
H-3 & 3.89041 & 3.87967 & $\mathbf{0 . 0 1}$ \\
H-4 & 3.50860 & 3.50700 & 0.001 \\
H-5 & 3.77780 & 3.76200 & $\mathbf{0 . 0 1 5}$ \\
H-6 & 3.80110 & 3.79999 & 0.001 \\
\hline
\end{tabular}

Tabela 2. Deslocamentos químicos $(\delta)$ dos prótons de $\beta-C D$ complexada com cânfora em $D_{2} \mathrm{O}$

\begin{tabular}{cccc}
\hline Próton & $\boldsymbol{\beta}-\mathrm{CD}$ & $\boldsymbol{\beta}-\mathrm{CD} /$ Cânfora & $\boldsymbol{\Delta}$ \\
\hline H-1 & 4.99060 & 4.99690 & 0.01 \\
H-2 & 3.56900 & 3.58300 & 0.01 \\
H-3 & 3.89041 & 3.83670 & $\mathbf{0 . 0 5}$ \\
H-4 & 3.50860 & 3.51820 & 0.01 \\
H-5 & 3.77780 & 3.73310 & $\mathbf{0 . 0 4}$ \\
H-6 & 3.80110 & 3.80330 & 0.00 \\
\hline
\end{tabular}

O experimento de ROESY para o complexo de inclusão de cânfora/ $\beta$-ciclodextrina revelou que os hidrogênios $\mathrm{H}-3$ e $\mathrm{H}-5$ interagem com as metilas da cânfora em $\delta_{H}$ $0,94,0,96$ e $0,83 \mathrm{ppm}$. Além disso, as intensidades das correlações em $\mathrm{H}-3$ são mais fortes que em $\mathrm{H}-5$, o que pode sugerir uma maior interação com o $\mathrm{H}-3$, e, a partir disso, pode-se inferir que as metilas em questão não entram totalmente na cavidade da $\beta$ - ciclodextrina. Já para o complexo de inclusão de 1,8-cineol/ $\beta$-ciclodextrina, o experimento de ROESY mostrou que a correlação mais forte foi observada entre o $\mathrm{H}-3$ da $\beta$-ciclodextrina em interação com as metilas em $\delta_{H} 1,30$ ppm e em interação com a metila em $\delta_{H} 1,12$ ppm. Essas informações podem auxiliar na interpretação do modo como a inclusão ocorre. 

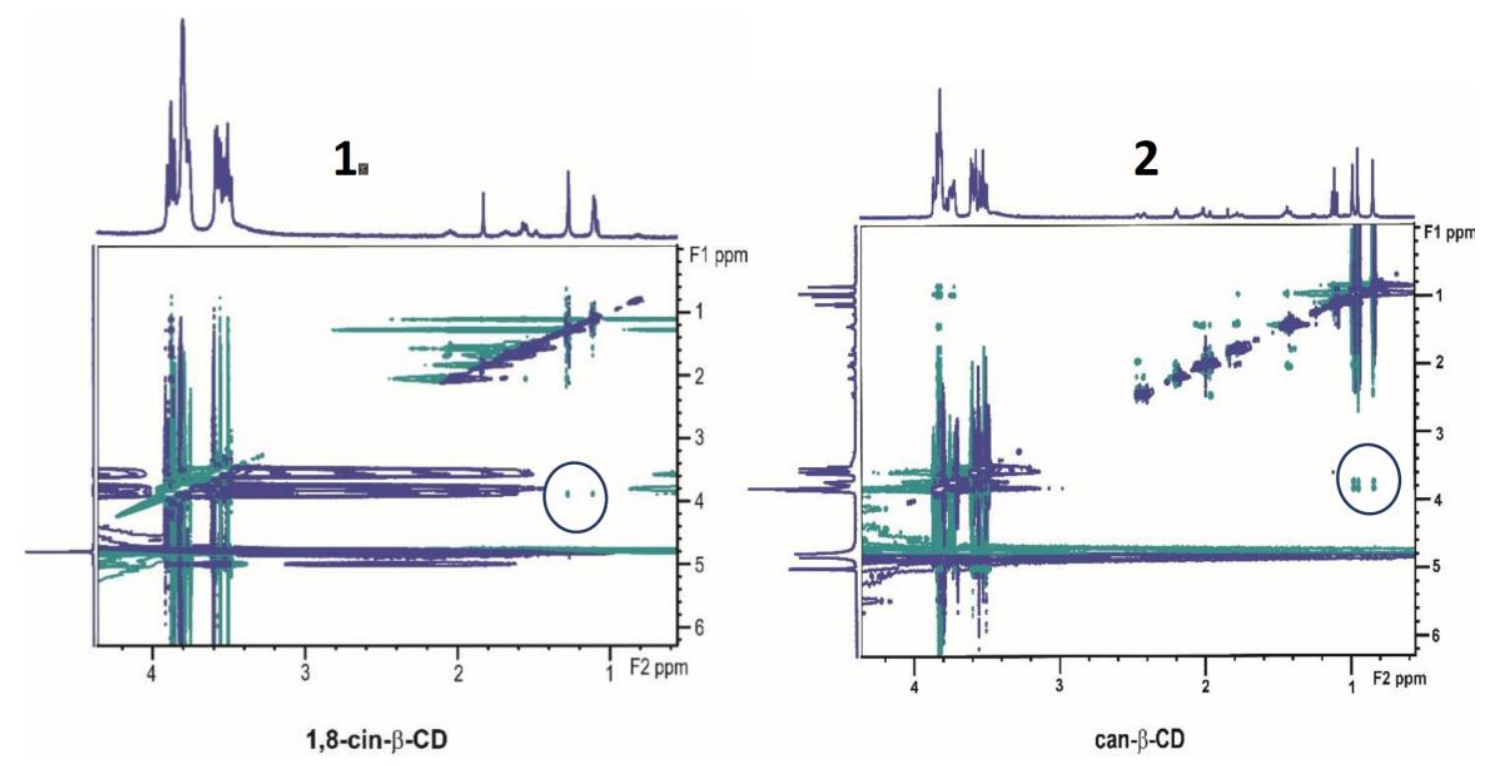

Figura 6. Experimento ROESY a $25^{\circ} \mathrm{C}$ em $\mathrm{D}_{2} \mathrm{O}$ para (1) o complexo de inclusão de 1,8-cineol em $\beta-C D$ e (2) o complexo de inclusão de cânfora em $\beta-C D$

\section{Conclusão}

A caracterização físico-química permitiu concluir que houve a formação dos complexos de inclusão de cânfora e 1,8-cineol em $\beta$ ciclodextrina. Dentre as técnicas utilizadas, a ressonância magnética nuclear se mostrou a mais vantajosa, uma vez que mostra claramente se a molécula hóspede está inserida na $\beta$-ciclodextrina através da análise da variação dos deslocamentos dos hidrogênios no interior da cavidade. Os resultados foram importantes para complementar os estudos na área de tecnologia farmacêutica, uma vez que os complexos podem aumentar a solubilidade e diminuir a volatilidade dos monoterpenos, podendo auxiliar na sua manipulação e avaliação da ação farmacológica.

\section{Referências Bibliográficas}

${ }^{1}$ Vianna, G. S. B.; Leal, L. K. A. M.; Vasconcelos, S. M. M.; Plantas medicinais da Caatinga: atividades biológicas e potencial terapêutico, 1a. ed., UFC: Ceará, 2013.
${ }^{2}$ Randau, K. P.; Florêncio, D. C.; Ferreira, C. P.; Xavier, H. S. Pharmacognostic study of Croton rhamnifolius HBK and Croton rhamnifolioides pax \& hoffm. (Euphorbiaceae). Revista Brasileira de Farmacognosia 2004, 14, 89. [CrossRef]

${ }^{3}$ Silva, J. S.; Sales, M. D.; Gomes, A. D. S.; Carneiro-Torres, D. S. Sinopse das espécies de Croton L. (Euphorbiaceae) no estado de Pernambuco, Brasil. Acta Botanica Brasilica 2010, 24, 441. [CrossRef]

${ }^{4}$ Gupta, V. K.; Natural products: research reviews, vol 1, Daya Publishing House: New Delhi, 2012.

${ }^{5}$ Ramos, J. M. O.; Santos, C. A.; Santana, D. G.; Santos, D. A.; Alves, P. B.; Thomazzi, S. M. Chemical constituents and potential antiinflammatory activity of the essential oil from the leaves of Croton argyrophyllus. Revista Brasileira de Farmacognosia 2013, 23, 644. [CrossRef]

${ }^{6}$ Almeida, J. R. G. S.; Souza, A. V. V.; Oliveira, A. P.; Santos, U. S.; Souza, M. D.; Bispo, L. P.; Turatti, I. C. C.; Lopes, N. P. Chemical composition of essential oils from the stem barks of Croton conduplicatus (Euphorbiaceae) native to the Caatinga biome. African Journal of Pharmacy and Pharmacology 2015, 9, 98. [CrossRef] 
${ }^{7}$ Almeida, J. R. G. S.; Souza, A. V. V.; Oliveira, A. P.; Santos, U. S.; Souza, M. D.; Bispo, L. P.; Turatti, I. C. C.; Lopes, N. P. Chemical composition of essential oils from Croton conduplicatus (Euphorbiaceae) in two different seasons. Journal of Essential Oil Bearing Plants 2014, 17, 1137. [CrossRef]

${ }^{8}$ Oliveira-Júnior, R.G.; Ferraz, C. A. A.; Silva, J.C.; Oliveira, A. P.; Diniz, T. C.; Silva, M. G.; Quintans-Júnior, L. J.; Souza, A. V. V.; Santos, U. S.; Turatti, I. C. C.; Lopes, N. P.; Lorenzo, V. P.; Almeida, J. R. G. S. Antinociceptive effect of the essential oil from Croton conduplicatus Kunth (Euphorbiaceae). Molecules 2017, 22, 900. [CrossRef]

${ }^{9}$ Oliveira-Júnior, R. G.; Ferraz, C. A. A.; Silva, J. C.; Teles, R. B. A.; Silva, M. G.; Diniz, T. C.; Santos, U. S.; Souza, A. V. V.; Nunes, C. E. P.; Salvador, M. J.; Lorenzo, V. P.; QuintansJúnior, L. J.; Almeida, J. R. G. S. Neuropharmacological effects of essential oil from the leaves of Croton conduplicatus Kunth and possible mechanisms of action involved. Journal of Ethnopharmacology 2018, 221, 65. [CrossRef]

${ }^{10}$ Quintans-Junior, L. J.; Araujo, A. A. S.; Santos, M. R. V.; Bonjardim, L. R.; Quintans, J. S. S.; Silva, J. C.; Nunes, X. P.; Almeida, J. R. G. S. Em: Recent Progress in Medicinal Plants Vol. 38: Essential Oils III and Phytopharmacology, 38th ed, Govil, J. N.; Bhattacharya, S. (Org.). Studium Press: Houston, 2013, cap.6.

${ }^{11}$ Cunha-Filho, M. S. S.; Sá-Barreto, L. C. L. Utilização de ciclodextrinas na formação de complexos de inclusão de interesse farmacêutico. Revista de Ciências Farmacêuticas Básica e Aplicada 2007, 28, 1. [link]

12 12. Marques, H. M. C. A review of cyclodextrin encapsulation of essential oils and volatiles. Flavour and Fragrance Journal 2010, 25, 313. [CrossRef] 